quent, à une première oscillation de vilesse, alleignant te maximum et le dépassant dune quantité à déterminer, succédera, obligatoircment, une marche à admission constante suivant la caractéristiquc mécanique définitive à utiliser.

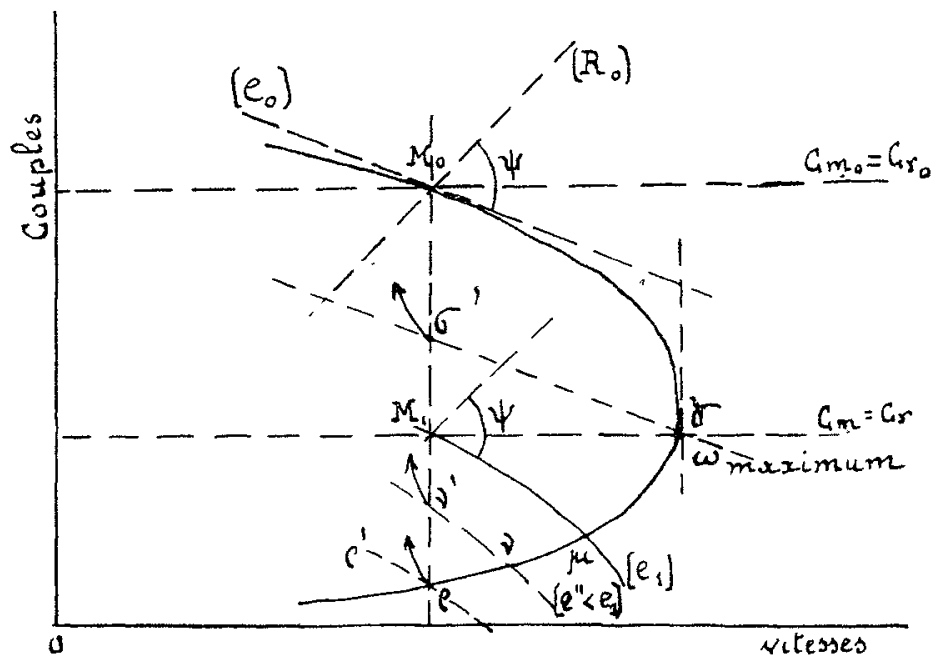

Fig. 4. - Réglage parfait avec criterium mixte (tachymètre et accélëromètre).

of' Coupure au maximum de vitesse (Régulateur accélérométrique) oscillations nouvelles $e^{\prime}>e_{1}$.

pu. Réglage parfait sur admission définitive $e_{1}$.

$\mu \mathrm{M}_{1}$ Régulateur asservi; mais réglage imparfait; nouvelles oscillations en $v^{\prime}$.

$q q^{\prime}$ Coupure à la fin d'une oscillation complète. Régulateur à tachymètre non asservi. Nouvelle oscillation cn p'.

N. B. - Pour simplifier, on a supposé le régulateur pảrfaitement isochrone (ou compensé) et parfaitement sensible.

Par ce seul exemple, de prédélermination d'unc perturbation sur diagrammes, suivie logiquement de lá réalisation d'un régulateur qui semble donner d'cxcellents résultals', on pourra induire quels fruclueux avantages découlent dans les domaines analogues d'études théoriques en apparence désintér'essées, bases indispensables néanmóins à l'établissement d'apparcils pratiquement satisfaisants.

El, du reste, n'en est-il pas de même dans tous les ordres de sciences et de travaux ? La télégraphic sans fil n'a-t-elle pas découlé d'expériences de laboraloires, faites sur la variabilité de résistance de tubes à limaille, sous l'influence dés oscillations hertziennes ?. Nos industricls n'appliquent-ils pas scicmment (nous ne leur ferons pas l'injure de croire qu'ils le font inconsciemment) les lois d'Ohm et de Joule ? Heureux sont-ils de n'avoir pas à se procurer pour cet emploi le bénéfice d'une licence !

BARBILIION,

Directcur de l'Iristilut Polytechnique de Grenoble.

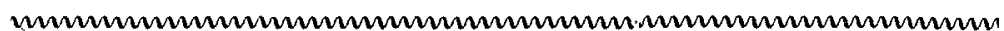

\section{NOTE DE LA RÉDACTION}

Nos lecteurs trouveront en dernière page le compte rendu de la Fête donnée par la Société "La Houille Blanche " en l'honneur de la vingtième année d'enseignement et la nomination dans l'ordre de la Légion d'honneur de $M$. le Professeur Barbillion.

Qu'il nous soit permis, en cetie occasion, d'adresser à ce Maitre l'hommage de notre respectueuse admiration.

\section{GONSTRUGTION}

ET

\section{Applications de Dispositifs de Barrages Anlomatiques (SUITE ET FIN)}

Dans les rivières qui charrient relativement peu d'alluvions ou dans les barrages où les parties mobiles reposent sur un seuil fixe relatirement élevé et où des vannes de fond sont prévues pour l'évacuation aes dépôts de sable et graviers, on utilisera également des clapets automatiques du type représenté par les fig. 3 et 18-19. Actuellement plusieurs barrages

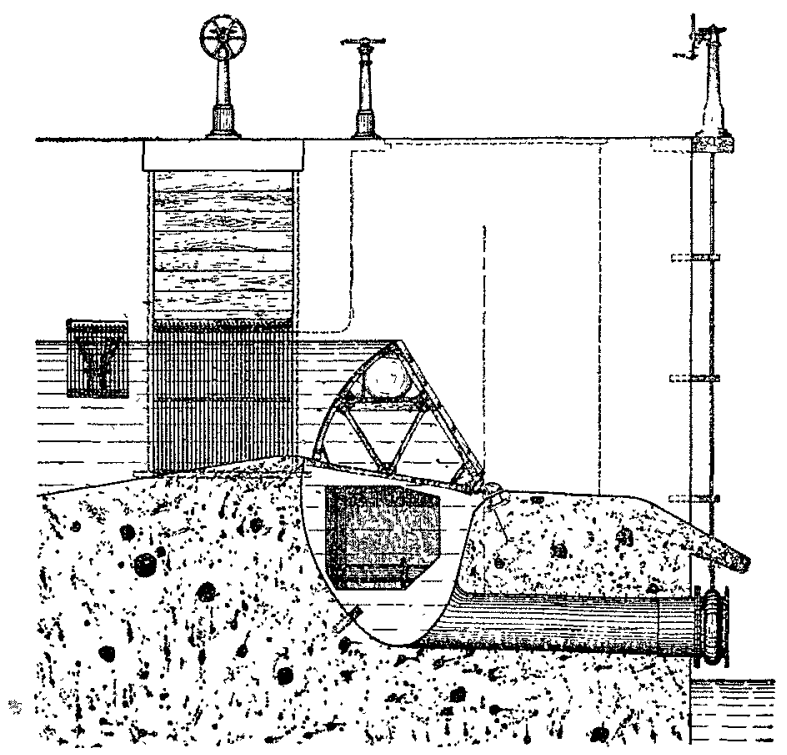

Fig. 18. - Vanne aulommlique a sectour su ln Vienne inforieure (France)

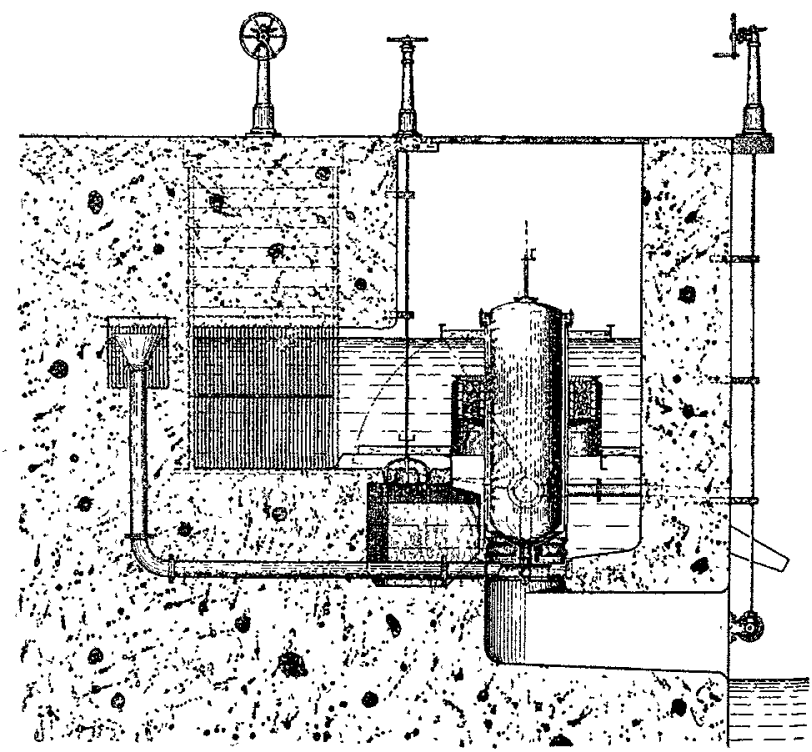

Fıg. 19. - Vanne automatique à secteur sur la Vienne infórieure (France)

automatiques importants de césystème sont en construction sur la Romanche (Isère), et sur le cours inférieur de la Vienne. Cette construction présente l'avantage de fonctionner tout à fait librement sans être influencée par le reflux venant du bief inférieur et de laisser le profil de la rivière au dessus de l'eau entièrement libre. De plus, le barrage se laisse abattre et relever de la rive sans peine par un seul homme en quelques minutes. Pour éviter des dépôts de sable et de vase dans les chambres, il est prévu des conduites et des vannes de purge, qui restent constamment ouvertes pendant l'afflux des eaux, 
mais ne sont utilisées que de temps à autre pendant la période des basses eaux.

Le barrage actuellement en construction à Livet, sur le cours de la Romanche, comprend deux clapets automatiques du

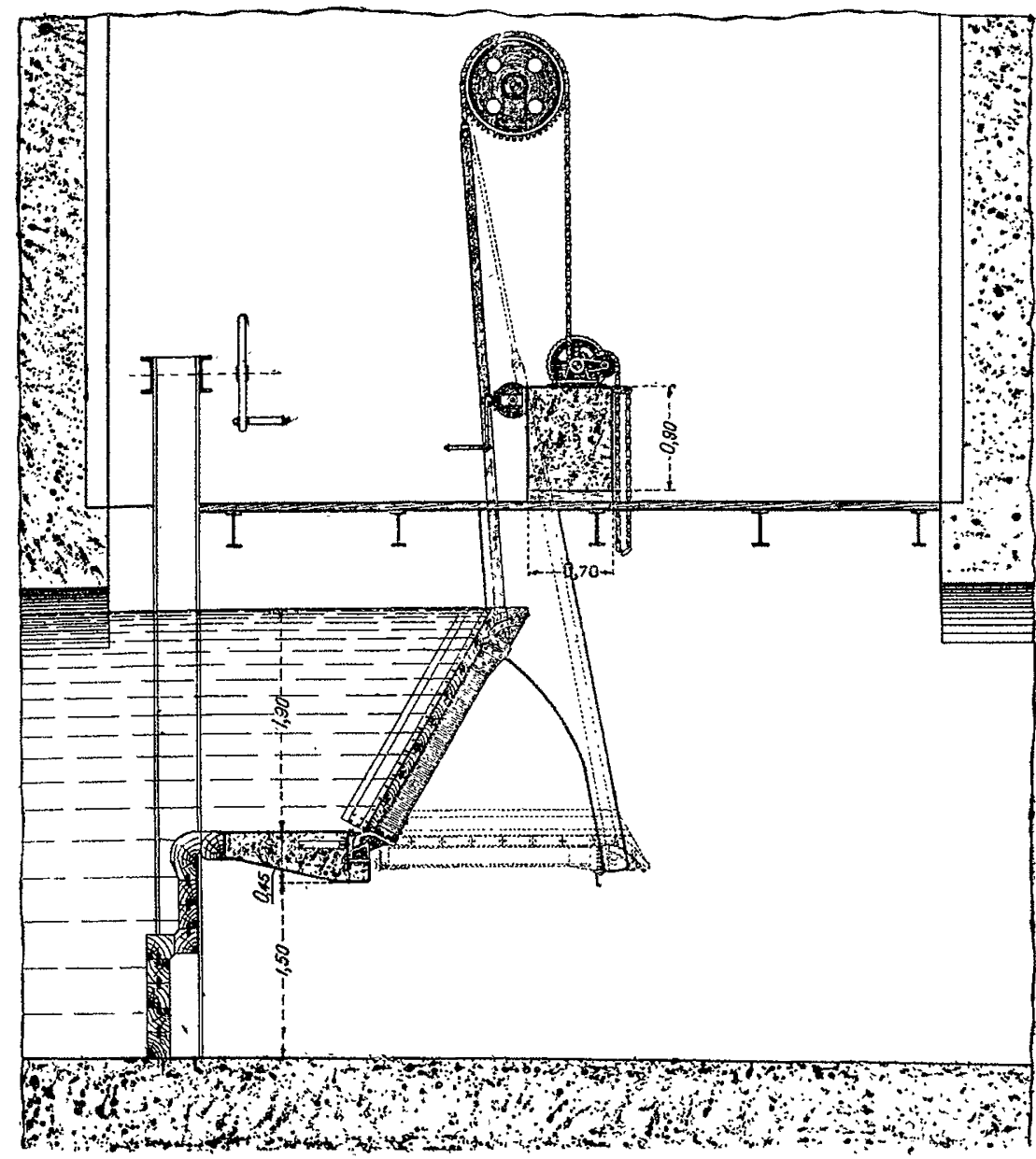

Fis. 20. - Canpetaderesoir auhomatique of à commande a main, combence aves ume ramne a glissieves fommat vane de fond. les clapets de s'abaisser entièrement. Les chambres des contrepoids sont pourvues de déversoir à crête réglable, par dessus laquelle l'eau d'amont pénètre dans les chambres de contrepoids en allégeant ceux-ci et en obligeant ainsi les clapets a s'abaisser complètement. Cette disposition permet d'abaisser facilement les clapets à volonté, en ourrant simplement une vanne d'entrée d'eau dans les chambres des contrepoids.

Le mouvement des rannes à secteur est obtenu par la variation de la pression hydraulique sous le secteur, au moyen de la vanne cylindrique automatique brevetée (Fig. 19).

La construction d'un barrage hydraulique de grandes dimensions, suivant le type de la fig. 3 , va être entreprise incessamment sur le cours de la Vienne. Il comprend un déversoir de $70 \mathrm{~m}$. de longueur, divisé en deux clapets de $23 \mathrm{~m}$. et un clapet de $24 \mathrm{~m}$. de longueur, sans piles intermédiaires, avec une hauteur d'eau de $3 \mathrm{~m}$. 60 , et une vanne de fond de $2: 3 \mathrm{~m}$. de longueur et de $4 \mathrm{~m} .50$ de hauteur d'eau.

Les fig. 15-17 représentent un type de barrage automatique spécialement approprié aux cours d'cau charriant beaucoup de sable et de gravier. Ce système n'exige pas de maçonnerie considérable au-dessus du radier, et est le seul type de barrage fonctionnant librement, même dans le cas d'un radier absolument horizontal, ne présentánt pas de chute. L'appareil de bouchure, contrairement à celui des types décrits jusqu'ici, n'est pas un clapet, mais une vanne en forme de segment dont les supports sont placés au-dessus du niveau de l'eau tel qu'il en a dejà été utilisé avec succès en Suisse pour la construction de barrages (par exemple sur la Sarine, près de Fribourg). Mais tandis que dans les constructions connues jusqu'alors la vanne mobile était manceuvrée de la passerelle de service à l'aide de treuils, les appateils des fig. 15-17 sont à fonctionnenem . nt entièrement automatique, entrainant la suppression des treuils et des passerelles. Le fonctionnement automatique s'obtient à l'aide de contrepoids montés sur les bras reliant la vanne à ses axes d'articula-

type de la fig. 3 , de $14 \mathrm{~m}$. 20 de largeur et $2 \mathrm{~m} .40$ de hauteur effective, chacun séparé par une pile intermédiaire. Ils sont commandés séparément par deux syphons disposés dans les piles de rive. Le caractère torrentiel de la Romanche et ses apports de sable et de gravier ont conduit à munir chacun des clapets d'une vanne de purge des chambres de pression. s'ouvrant automatiquement aussitôt que le niveau d'amont dépasse de $2,3 \mathrm{~cm}$. la hauteur de retenue normale et restant ouverte aussi longtemps que le clapet n'a pas repris sa position initiale. Ces rannes de purge peurent être mancuv rées aussi à volonté à l'aide d'un treuil. I'installátion offre ainsi toute sécurité de fonctionnement.

Le barrage en cours d'exécution sur la Vienne, pour le compte de la Manufacture nationale d'Armes de Chatellerault, comprend cinq vannes dont trois clapets analogues à celui de la fig. 1 , de $14 \mathrm{~m} .50$ et $12 \mathrm{~m} .85$ de largeur, et de $3 \mathrm{~m}$. de hauteur effective, et deux vannes à secteur commandées par vanne cylindrique automatique (fig. 18 et 19), de $19 \mathrm{~m} .75$ de largeur et $1 \mathrm{~m} .15$ de hauteur

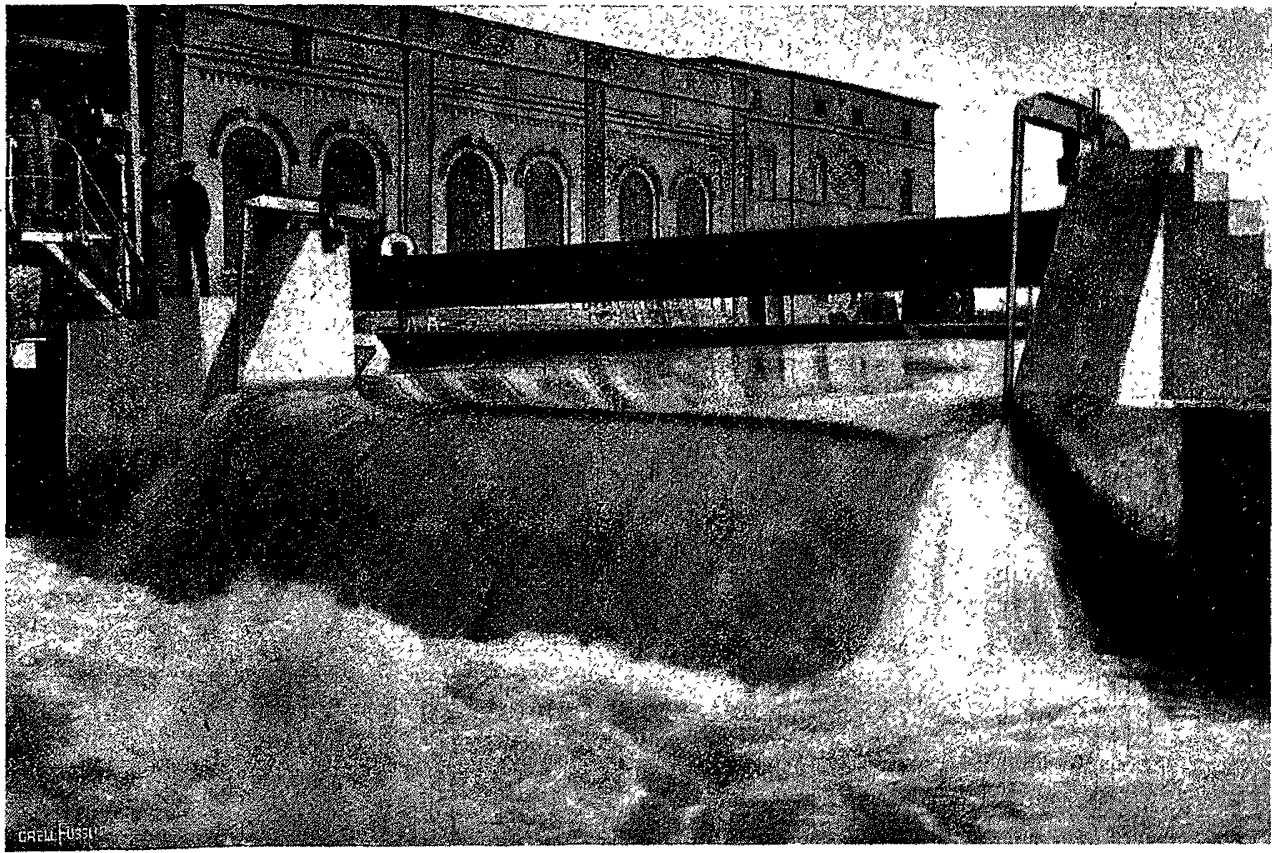

Fig. 21. - Clapet-devensoin formant pertuis de dicharge automatique daus le onnal d'amence de la filature du Sladtbach à Augsbourg (Baviore). d'eau chacune. Les trois clapets de $3 \mathrm{~m}$. do hauteur'd'eau sont munis chacun de deux contrepoids en béton armé se déployant dans des chambres aménagées dans les piles latérales. Cette disposition a été adoptée pour compenser l'influence du reflux d'aval, qui empêcherait tion. La première paire de contrepoids tendant à ouvrir la vanne ont une valeur constante, tandis que la deuxieme paire tendant à la fermer, disposée dans des chambres allongées, sur les côtés du canal, subissent une poussée 


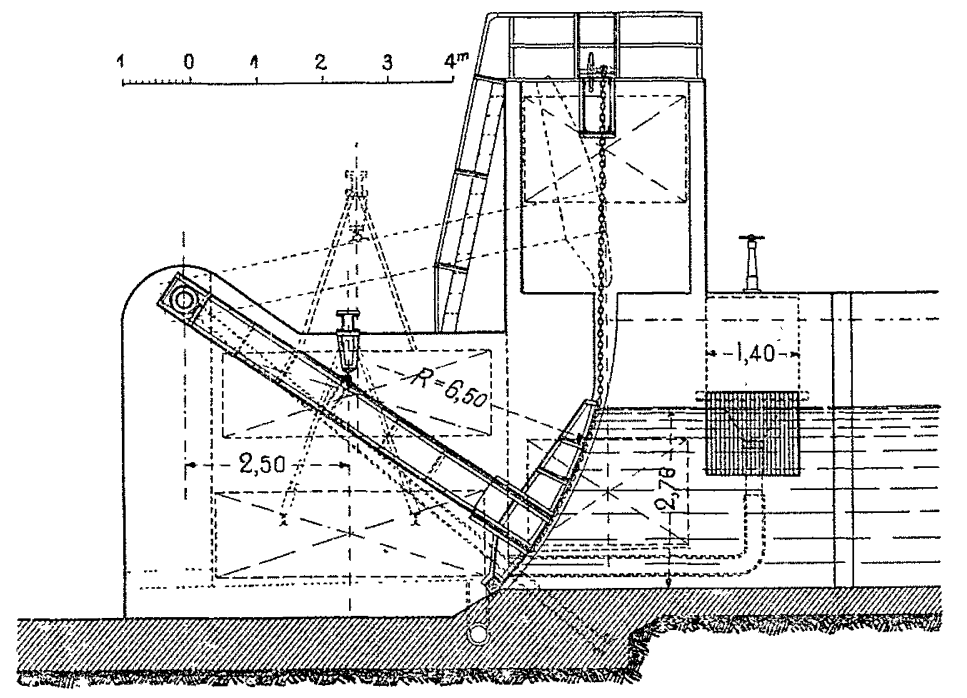

Fig. 22. - Vanne aulomalique et à commande à main prévue pour un barrage sur la Reuss.

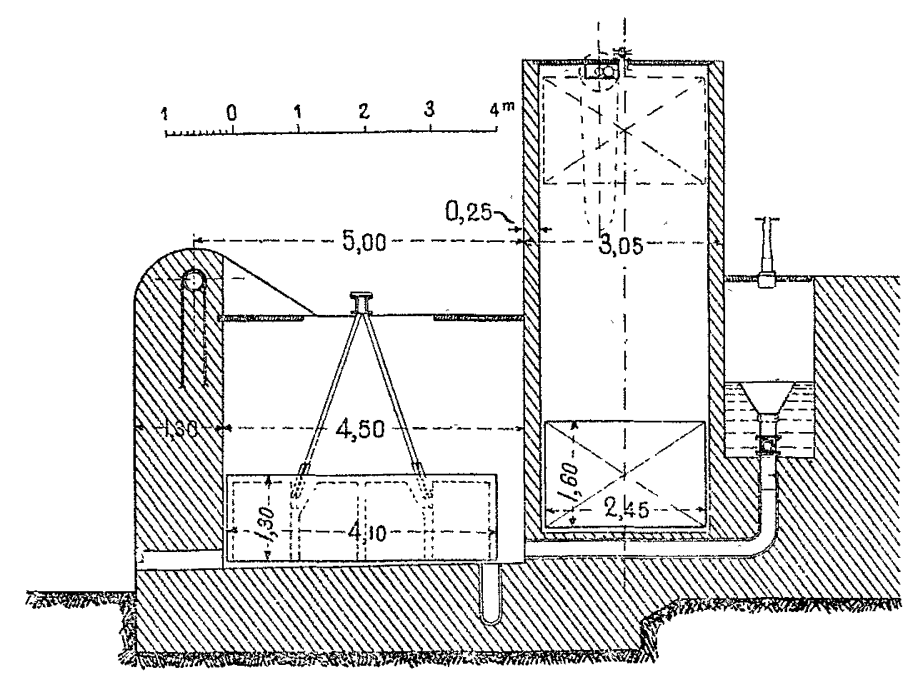

Fig. 23. - Vanne aubomalique et à commande à main, prévue pour un barrage sur la Reuss.

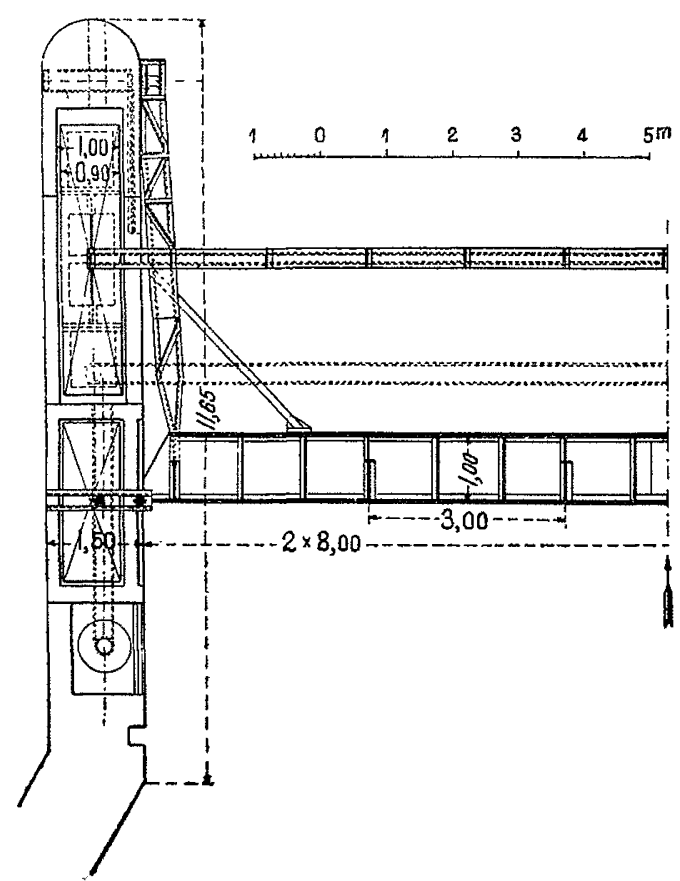

Fig. 2\%. - Vanne automatique et' à commande à main, prévue pour un barrage sur la Reuss.

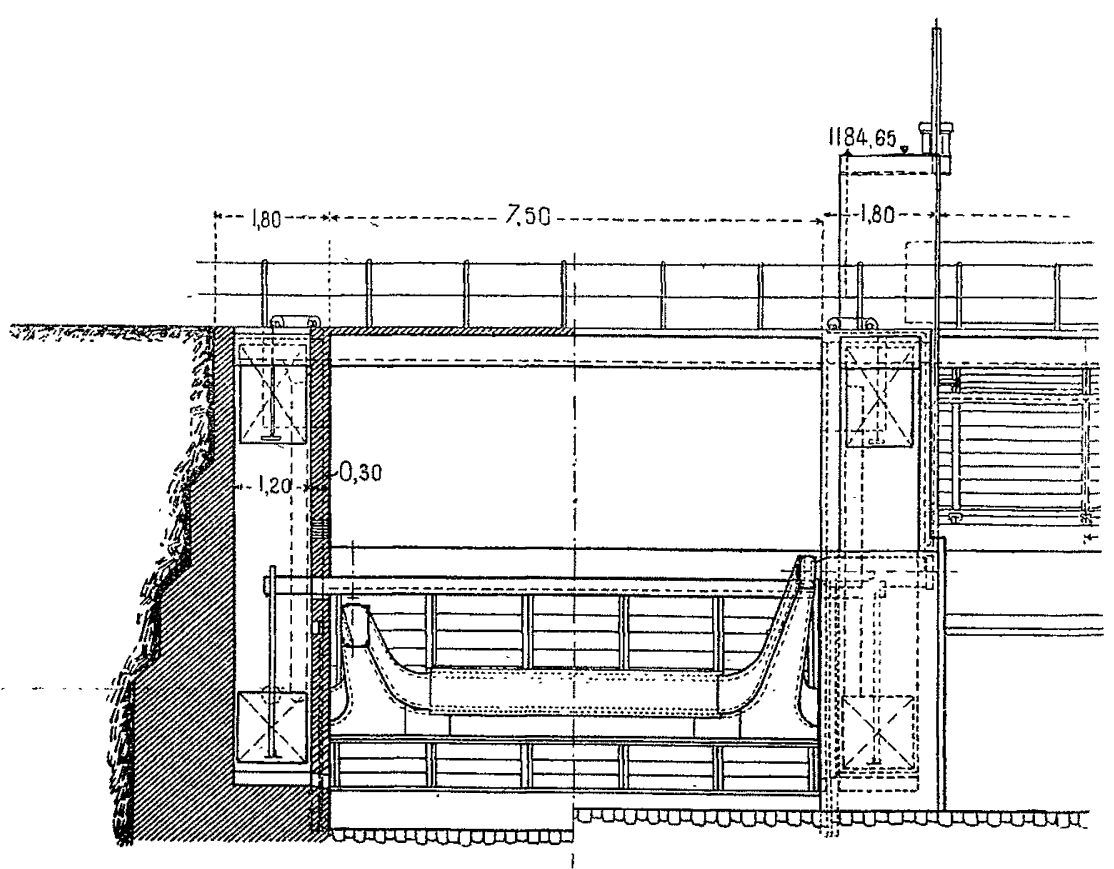

Fig. 26. - Vanne de fond autamatique avec commiande à main, pour un bassin rlírcumulation, adjacente à un clapet-déversoir à contrepoids supertieur de $12 \mathrm{~m}$. de largeur.

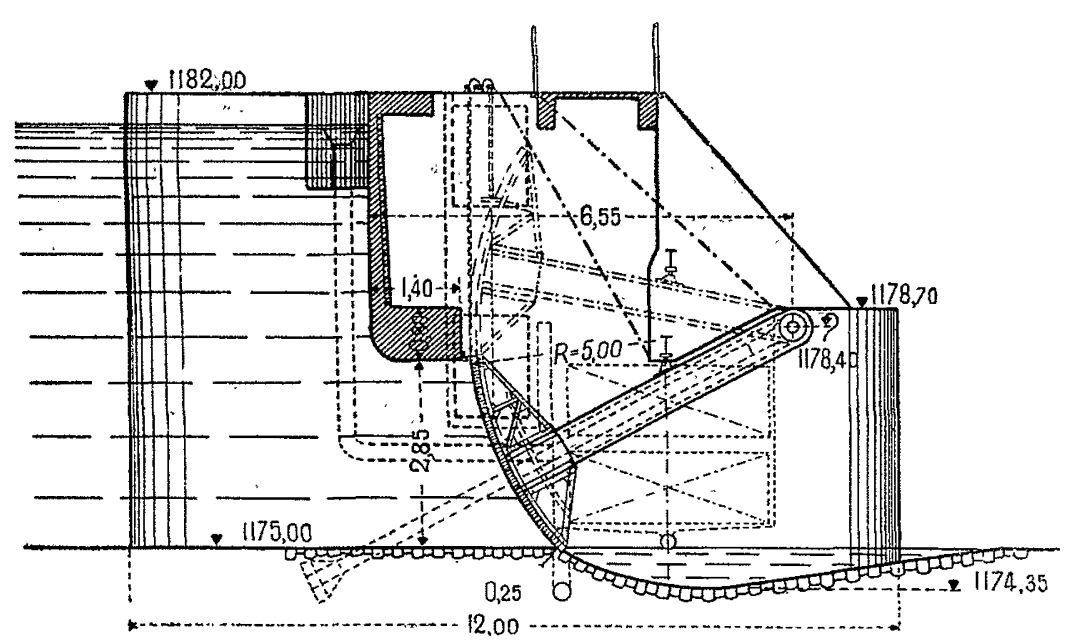

Fig. 25. - Vanne automatique et à commande a main, servant de vanne de fond pour un bassin d'accumulation.

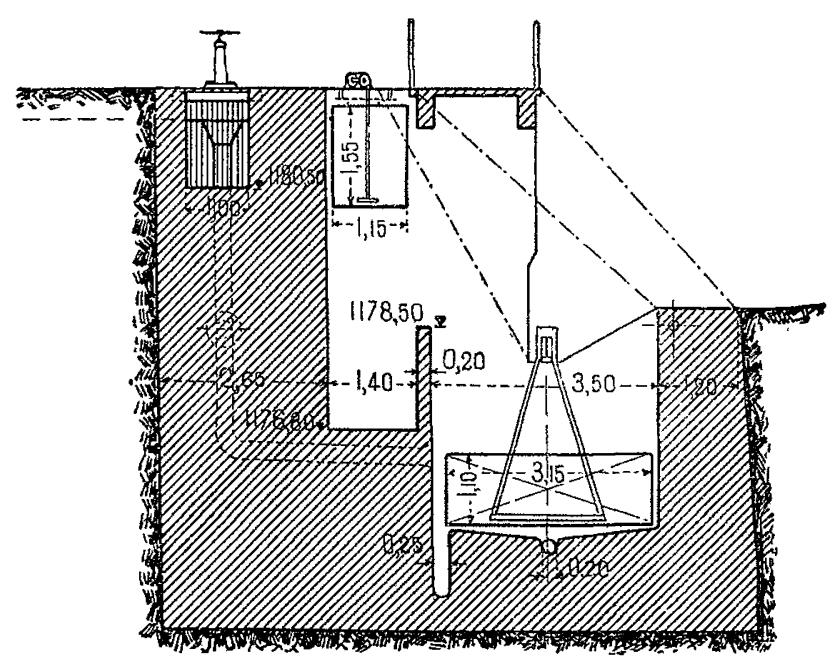

Fig. 27. - Vanne de fond automalique avec commande à main. pour un bassin diaccumulation. 
dont l'intensité est en rapport avec la cote du niveau d'amont aussitôt que la rețenue normale se trouve dépassée. Ici, également, le réglage à un niveau constant en présence d'un afflux variable, s'opère simplement par l'action réciproque des

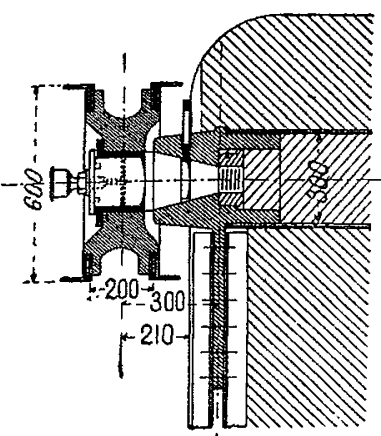

Fig. 27 a. - Yanne de fond aulomatique avec commande à maiı, pour un bassm daccumulation. Axe diarticulation

le niveau des plus hautes eaux. Elle demeure dans cette position jusqu'à ce que le niveau de l'eau ait atteint à-nouveau sa valeur normale. La vanne recommence alors à régler l'écoulement de l'eau dans la mesure où cela est nécessaire pour la maintenance de la retenue normale. Les fig. 16 et 17 représensentent une. vanne semblable installée dans la Thur près de Ebnat-Kappel, lés fig. 22-24, un projet de vanne automatique destinée à la transformation d'une installation existante sur la Reuss. Des raisons d'esthétique ont conduit à placer. dans cette dernière construction, les contrepoids de relevage non plus aux extrémités supérieures des leviers, mais dans des puits spécialement construits à cet effet dans les piles de rive. Outre la bonne évacuation des alluvions et le balayage énergique du radier, ce type d'appareil de bouchure automatique a l'avantage de posséder des articulations à l'air libre et de permettre une réparation facile du corps de la vanne une fois relevé. Les plus grands barrages de ce système peuvent également être manœuvrés de la rive sans effort par. un seul homme en admettant l'eau dans les chambres des contrepoids, comme c'est le cas des installations repréśentéés par les fig. 11-14. Cette manœuvre est indiquée lorsqu'il s'agit, dans des périodes prolongées de basses eaux, de balayer de temps à autre le radier, ou encore en cas de corrections éventuelles des berges.

\section{GROUPE III}

Vannes automatiques de décharge sur Canaux d'amente

$$
\text { D'USINES }
$$

Dans le troisième groupe, comprenant les canaux de prise (dispositifs de décharge automatiques) d'usines, les appareils de bouchure automatiques rivalisent avec les siphons-déversoirs. Pour des débits restreints, jusqu'à environ $5-6 \mathrm{~m}^{3}$ par seconde, ces derniers soutiendront la concurrence avec avantage, car leur coût, pour de petites dimensions, est inférieur à celui de clapets qui, pour réaliser un bon fonctionnement, doivent atteindre une grandeur déterminée. Pour des débits plus considérables de $6 \mathrm{~m}^{3}$ par seconde environ, les clapets automatiques prennent déjà le dessus, car non seulement (dans la plupart des cas), leur cout est inférieur ou tout au moins égal à celui des siphons de même capacité, mais encore ils écoulent l'eạu plus également vers l'aval (les siphons débitent subitement, au moment de l'amorçage, une puissante vague d'eau) et, lorsqu'ils sont pourvus de dispositifs de manœuvre, ils peuvent être utilisés pour la vidange du canal.

Tous lés systèmes d'appareils de bouchure automatiques décrits jusqu'ici ont déjà été employés comme organes de décharge pour canaux. L'une des constructions les plus fréquemment adoptées est représentée par la fig. 20. Le clapetdéversoir automatique y est combiné a vec une vanne à glissière ordinaire, qui sert de vanne de fond pour le balayage du radier et pour la vidange totale du canal, tandis que le clapetdéversoir qui la surmonte règle de lui-même le niveau et évacue la glace ou les aụtres corps flottants L'ensemble constitue en quelque sorte un perfectionnement de la vanne double généralement employée en pareil cas, dont on abaisse la partie supérieure qui est ici formée par le clapet automatique. Un treuil monté sur le contrepoids permet ici aussi d'abaisser le clapet à volonté.

La fig. 21 représente une disposition semblable construite dans le canal d'amenée des turbines de la filature de coton sur le Stadtbach à Augsbourg. La capacité est d'environ $20 \mathrm{~m}^{3}$ par seconde.

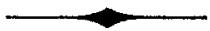

\section{GROUPE IV}

VANNES DE FOND AUTOMATIQUES POUR BARRAges MASSIFS

Le quatrième groupe comprenant les vannes de fond automatiques pour barrages fixes et bassins d'accumulation ne permet d'utiliser qu'un nombre plus restreint de constructions.

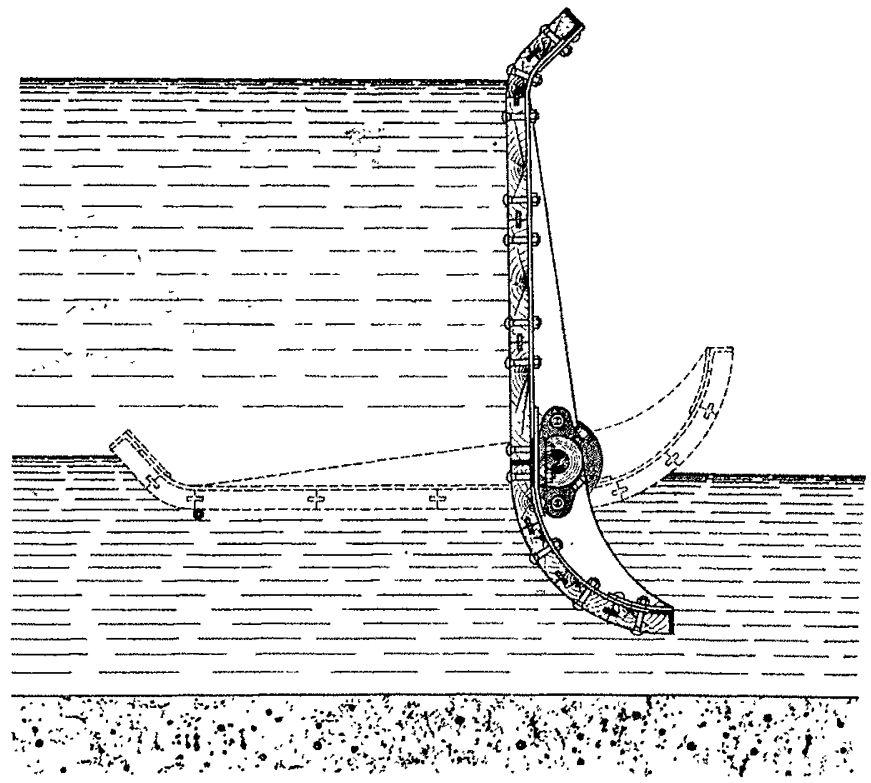

Fig. 28. - Clapet automatique régulatour de debit.

Il s'agit ici exclusivement du type représenté par les fig. $15-17$, soit la vanne automatique à segment. Les fig. 25-27 indiquent l'utilisation de ces appareils comme vanne de fond automatique complétée par un clapet-réservoir adjacent à contrepoids supérieur.

Cette combinaison permet d'écouler automatiquement sans élévation appréciable de la retenue, le volume entier des crues, et d'évacuer les glaçons et corps flottants (par le clapet) en même temps que de balayer efficacement le radier.

La vanne de fond étant munie du dispositif simple de manceu- 
vre dẹcrit plus haut, le bassin d'accumulation peut être vidé entièrement en tout temps. On a la possibilité, en chargeant plus ou moins les contrepoids, de faire fonctionner en premier lieu soit le clapet-déversoir, soit lá vanne de fond automatique, selon' que l'on estime indiqué de chassèr immédiatement les apports de sable et graviers au début de la crue, ou qu'on préfère utiliser le clapet-déversoir pour que le radier ne soit pas trop attaqué. teurs, ou bien on ménage des bassins de régularisation sur les côtés du canal de fuite. Les appareils automatiques suivants servent à régler uniformément l'écoulement de ces réservoirs, indépendamment du niveau momentané.

La fig. 28 représente un dispositif simple, qui est utilisé lorsque le volume d'eau à livrer reste à peu près le même. Lè clapet tourne autour d'un axe horizontal placé à environ $1 / 3$ de la hauteur. Cet axe suit, dans la plupart des cas, une courbe
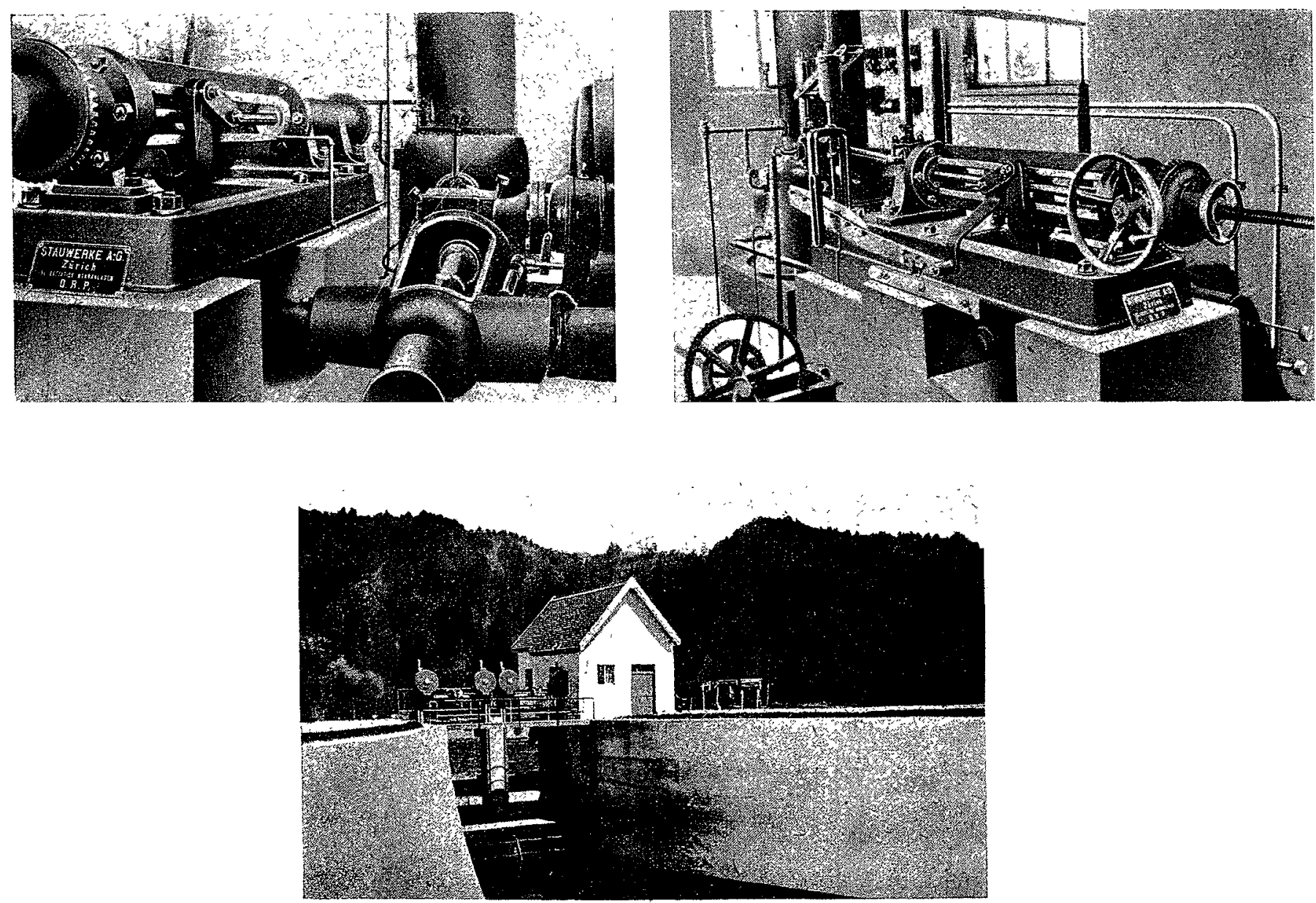

Fig. 29.31. -- Réglage aulomalique de déhit. fonctiomnanl dans les iimites de 3 a $15 \mathrm{~m}^{3}$ par seconde, sur le canal de fuite de l'usine de Leitzach (IIaule-Bavière).

\section{GROUPE V}

Dispositifs automatroues D'Écoulement livrant un volume D'EaU UNIFORME MalgRÉ LES VARIATIONS DE NIVEAU

Les appareils qui constituent le cinquième groupe ont un but essentiellement différent. Il s'agit ici d'écouler sous une pression variable une quantité d'eau fixée d'avance, qui peut être constante ou variable. Ce cas se présente fréquemment pour des usines de force accumulant l'eau par intervalles, tout en étant tenues de livrer aux usiniers suivants, pendant 24 heures, un volume d'eau uniforme. D'ordinaire les cánaux de fuite sont, - en pareil cas, transformés en bassins compensa- déterminée, et le clapet s'abaisse de sa position initiale jusqu'i la position horizontale, selon que le niveau d'amont est élevé ou s'abaisse.

La position du clapet est déterminée par l'équilibre des moments des pressions d'eau sur le bras supérieur et inférieur. Lorsque le niveau d'amont baisse, le clapet'se rapproche de la position horizontale, pour se relever quand ce niveau remonte.

En donnant une forme appropriée à la cóurbe décrite et au profil du bras inférieur, on réalise l'égalité de débit sous le clapet (ou sa variation suivant des lois déterminées), pour toutes les hauteurs d'eau.

Lorsque la quantité d'eau à livrer doit subir des variations plus sensibles, le simple dispositif décrit ci-dessus n'est plus applicable. En pareil ca's on utilise des vannes à segment 
mues par la pression de l'huile, qui sont commandées par des flotteurs différentiels dépendant des niveaux amont et aval. complétés par des appareils de contre réglage.

Les fig. 29-31 montrent un mécanisme de ce genre installé sur le bief inférieur de l'usine de Leitzach (Bavière), et construit pour fournir régulièrement une quantité d'eau que l'on peut faire varier de 3 à $15 \mathrm{~m}^{3}$ par seconde.

Le réglage des divers débits s'opère du tableau de distribution éloigné d'environ $2 \mathrm{~km}$., sur lequel le réglage momentané est signalé électriquement et s'inscrit automatiquement sur une échelle graduée. L'installation est en service depuis 1914 et fonctionne très exactement, comme il ressort du rapport d'expertise dressé lors de la 'réception (1).

\begin{tabular}{ccc} 
Hauteur d'eau & Débit prévu & Débit réel \\
- & - & 13,10 \\
3200 & 13,00 & 12,93 \\
3078 & 13,00 & 12,92 \\
2885 & 13,00 & 12,90 \\
2811 & $13, c 0$ & 13,00 \\
2720 & 13,00 & 12,90 \\
2572 & 13,00 & 13,05 \\
2442 & 13,00 & 13,15 \\
2310 & 13,00 & \\
\hline 2186 & 15,00 & 15,16 \\
1990 & 15,00 & 15,05 \\
1787 & $15, C 0$ & 15,00 \\
\hline 5700 & 8,00 & 8,14 \\
3000 & 8,00 & 7,95 \\
1000 & 8,00 & 7,97 \\
450 & 8,00 & 8,02 \\
\hline 450 & 3,00 & 3,92 \\
\hline 5000 & 3,00 & 3,10 \\
\hline
\end{tabular}

Relativement au coût des appareils de bouchure automatiques décrits ci dessus l'on peut dire que les frais d'achat ne sont en général pas plus élevés que ceux des vannes ordinaires à glissières ou des vannes Stoney. Les diverses formes d'exécution suivant lès'fig. 1-31 coûtent, même la plupart du temps, sensiblement moins cher que des vanneș à panneaux, avantage auquel vient s'ajouter le gain réalisé du fait de l'infrastructure plus simple, de la suppression des piles intermédiaires, etc.

Les frais d'entretien et de manœuvre sont nettement inférieurs à ceux que nécessitent des vannes à glissières ou à rouleaux, grâce à la suppression de la manœuvre, de la surveillance et du graissage; du fait de la simplicité de la construction, de la suppression de treuils, chaînes, câbles, etc., les réparations sont naturellement moins fréquentes.

La totalité des installations décrites ont été exécutées par la S. A. des Barrages Automatiques Zurich, et brevetées par cette Société, qui, depuis dix ans, s'est acquis la spécialité exclusive de la construction de ces appareils automatiques.

\section{E. Froté, Ingénieur}

(1') Communuqué par la Sociéte Anonrme des Burrages Automatiques de Zurich.
L'USURE

DES

\section{TURBINES HYMRAULIQUES}

\section{SES CONSÉQUUENCES ET LES MOYENS d'Y PARER}

\author{
(SÜTE ET FIN)
}

\section{IV}

\section{L'Usure des Turbines et le Dessableur de l'Usine de l'Ackersand.}

Une description détaillée de l'usine de l'Ackersand, sur la Viège de Saas, en Valais, a été publiée par la Schweizerische Bauzeitung en I 909 ; nous la supposons connue aussi des lecteurs du Bulletin technique. Elle dispose d'une chute utile d'environ 7oo mètres et a été préruc pour quatre turbines de $5.500 \mathrm{HP}$ chacune, avec une cinquième comme réserve.

Les deux premières turbincs installées, furent longtemps seules en service $\mathrm{cl}$, grâce au dessableur de la prise d'eau à Saas-Balen, qui éliminait une grande partic des allurions contenues dans l'eau, les distributeur's des turbines n'étaient remplacés qu'une fois par an et les roues motrices duraient plusieurs années. Le fonctionnement de ce dessableur, dont la fig. 16 donne une vue extérieure, était donc considéré comme satisfaisant.

Depuis quelques années, l'équipement de l'usine est de quatre turbines visibles sur la fig. I 7 et la puissance des groupes, portée à $6.000 \mathrm{HP}$ chacun. Le débit utilisé a donc été plus que doublé et dépasse actuellement celui admis lors de la construction dı dessableur.

Malgré la grande vitesse de l'eau dans les deiux canaux de décántation qu'il comporte, le dessableur éliminait encore, sous ce nouveau régime, une très forte quantité d'alluvions. Le lriagre des dépôts trouvés dans les canaux de décantation; dont on avait enlevé les parois-guides transversales, fit voir, par exemple, que l'échantillon prélevé à 6 mètres de l'entrée contenait déjà le $17 \%$ en volume, de grains avec un diamètre inférieur à $\mathrm{x}, \mathrm{x} \mathrm{mm}$. et qu\% celui prélevé à 2 I mètres de l'entrée en contenail le $98 \%$. L'évacuation des dépôts au moment voulu, au moyen de "chasses". exigeait toutefois bcaucoup d'attention, du temps et du travail et portait préjudice à la décantation dans le seul des deux canaux restant en service. Après un certain tcmps, son action diminuait à tel point que l'on trouvait, dans les canaux de fuite des turbines, du sable en quantité, voire même des petits graviers allant jusqu'i r $2 \mathrm{~mm}$. de diamètre et que l'usure des tuŕbines prenail des proportions très inquiétantes.

Une augmentation du nombre des canaux de décantation aurait permis' d'améliorer cet état de choses, mais coûté fort cher et la perfection du dessablage scrait demeurée dépendante de l'attention du personnel. On comprend l'importance de ces facteurs, lorsqu'on sait qu'en rgrg, le dessableur transformé a éliminé el évacué jusqu'à 44 tonnes, et plus, d'alluvions par heure.

Après quelques études préliminaires; la "Société des Usines Electriques de Ia Lonza ", propriétaire de l'usine, adopta notre proposition tendant à un meilleur dessablage de l'eau par l'installation d'un dispositif de purge automatique et conlinue pour les alluvions éliminées, combiné avec une meilleure répartition et tranquillisation de l'eau dans les deux canaux de décantation. Ce nouveau principe de dessablage est breveté en Suisše et a l'étranger. Les travaux de transformation furent exécutés pendant l'hiver rgr 8 -rgrg.

Le dessableur, tel qu'il est aujourd'hui, est représenté par la fig. I 8. La transformation a consisté : dans le placement à l'entrée dì dessableur d'une séric de deux grilles en bois à fort ćcartement, d'un gouvernail pour la tranquillisation et la répartition du débit en parties égales dans les deux canaux, puis de deux séries de grilles à écartèment plus faible, dont la fonction est de tran- 